\title{
APPLICATIONS OF CROSS-CORRELATION SIGNAL RECOVERY IN PHOTOACOUSTICS
}

\author{
G.F. Kirkbright, R.M. Miller and A. Rzadkiewicz \\ Department of Instrumentation and Analytical Science, UMIST, P.O. Box 88, \\ Manchester M6O 1QD, U.K.
}

Résumé - La technique d'autocorrélation avec modulation pseudo aléatoire de la lumière incidente permet l'amélioration de l'analyse en profondeur de l'êchantillon en spectroscopie et en imagerie.

Abstract - The technique of cross correlation signal recovery with PRBS modulation of the incident iight allows improved depth profiling of samples in both spectroscopic and imaging applications.

\section{INTRODUCT ION}

One of the most attractive features of photoacoustic techniques is the ability to obtain depth related information from a sample. This ability has been used in both photoacoustic spectroscopy (1) and in thermal wave imaging $(2,3)$ to provide a more complete description of the three-dimensional characteristics of a sample than can be obtained using other techniques. The depth profiling ability arises because the absorption of light is not detected instantaneously by a decrease in transmitted or reflected light, but after a time delay during which the heat generated by light absorption diffuses through the sample, interacting with the physical structures of the material. Depth profiling allows photoacoustic techniques to be used for the non-destructive testing of a wide range of materials and sample types.

Conventionally, depth profiling has been achieved by controlling the frequency at which the incident radiation is modulated. At high modulation frequencies the thermal diffusion length is smal1, and the photoacoustic signal is obtained from a thin layer at the surface of the sample. At lower modulation frequencies the thermal diffusion length increases and a thicker layer of the sample can be examined. Within any modulation frequency range analysis of the phase angle of the signal can provide further depth information $(1,3)$. However, due to the limitations of single frequency modulation techniques this depth information is difficult to obtain, and even more difficult to analyse. Because only one modulation frequency is present at a time, a sample which absorbs light throughout its structure or consists of a number of overlapping layers gives rise to a thermal wave response which is the vector sum of a number of sine waves of different phase and amplitude. It is not possible to deconvolute the different components of such a response by measuring at one frequency. Full characterisation of a complex system requires measurement of the response at a large number of different frequencies. If the different modulation frequencies are applied sequentially this becomes a very time-consuming process. In addition the results are presented as a gain phase diagram which it is difficult to relate to the physical characteristics of the sample.

A more direct approach is to measure the impulse response of the sample using a multifrequency test signal. The simplest method would be to use an impulse perturbation signal and record the time-resolved photoacoustic response to the perturbation. However, to obtain good time resolution in the sample impulse response it is necessary to use an impulse which is short with respect to the response time of the sample. This implies that the source duty cycle is very low, and the average power absorbed by the sample is also low. To overcome the 
poor signal-to-noise ratio of the photoacoustic response, repeated experiments and ensemble averaging of the results would be required. An alternative is to use a multifrequency noise source together with correlation signal recovery. Cross-correlation signal recovery has been applied to both photoacoustic spectroscopy and thermal wave imaging $(5,6)$. This paper presents some recent results in this area.

\section{THEORY}

Two correlation functions can be defined which express the degree of similarity between a pair of signals as one is delayed by a time ' $t$ with respect to the other (4). The auto-correlation function, $R$, describes the degree of correlation for a single variable, $x(t)$, and the cross-correlation function $R_{x y}$ describes the correlation between two variables, $x(t)$ and $y(t)$.

$$
\begin{aligned}
& R_{x x}(\tau)=\frac{1}{T} \int_{0}^{T} x(t-\tau) \cdot x(t) d t \\
& R_{x y}(\tau)=\frac{1}{T} \int_{0}^{T} x(t-\tau) \cdot y(t) d t
\end{aligned}
$$

where $\mathrm{T}$ is the integration time and $\tau$ is the delay time. For a system perturbed by an input signal, $i(t)$, the input-output cross-correlation function, $R_{i z}$, is related to the system impulse response, $h(t)$, by the convolution integral

$$
R_{i z}(\tau)=\int_{0}^{T} s_{h(t) \cdot R_{i i}}(\tau-t) d t+R_{i n}(\tau)
$$

where $R_{i i}$ is the perturbation signal auto-correlation function, $R_{\text {in }}$ is the cross-correlation function for the perturbation signal and noise in the system and $T_{S}$ is the system settling time. If the test signal has the same autocorrelation function as an impulse function, then the input-output crosscorrelation function $R_{i}$ approximates to the system impulse response $h(t)$. Random noise is discriminated against by the small value of $R_{i n}$. The pseudorandom binary sequence (PRBS) is a test signal with suitable characteristics for correlation measurements. The PRBS test signal has the following characteristics: intervals defined by a clock period, $\lambda, t=0, \lambda, 2 \lambda, 3 \lambda \ldots \ldots$

$$
\text { At each time interval the probability of a level change is } 0.5 \text {. }
$$

The sequence of level changes is predetermined, allowing experiments to be repeated.

The sequence is periodic with period $\mathrm{T}_{0}=\mathrm{N} \lambda$, where $\mathrm{N}$ is an odd integer.

The PRBS has similar auto-correlation function to a pulse of width $\lambda$. If $\lambda$ is sufficiently small and $T_{0}$ is greater than $T_{s}$, the impulse response of the system can be measured. Since the PRBS has a duty cycle approaching $50 \%$ a substantial increase in signal-to-noise ratio and, therefore, a reduction in measurement time can be achieved.

\section{EXPERTMENTAL METHODS}

For correlation spectroscopy an existing single beam UV-Visible photoacoustic spectrometer (7) was modified by replacing the usual unity mark-space ratio chopper wheel with a chopper wheel with a 127 bit PRBS cut into the edge (5). The input modulation signal was monitored using a photodiode sensor inside the 


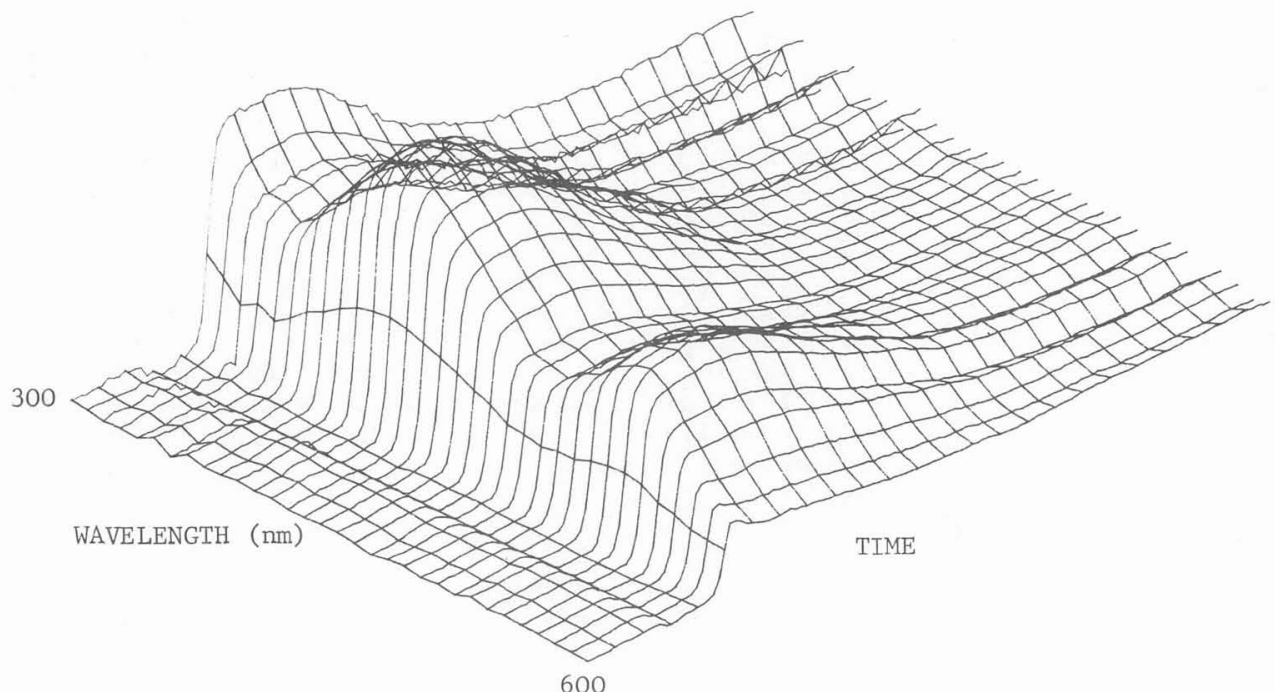

Figure 1. Cross Correlation Spectrum of a Bulk Dyed Red Polymer Film.
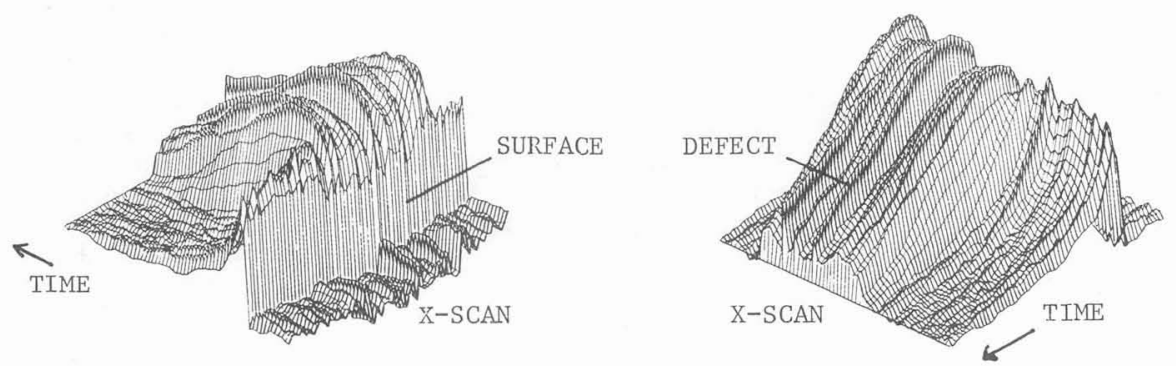

Figure 2. Cross Correlation Line Scan of an Opaque Sample with a Sub Surface Defect. 
monochromator housing. The PRBS period $T_{0}$ could be controlled by adjusting the chopper wheel rotation speed. The detector was a gas microphone photoacoustic cell (OAS401, EDT Research Ltd, U.K.). The photoacoustic signal from the cell was passed to a low-noise preamplifier (Model 4505, E.G. \& G.Brookdeal Ltd, U.K.) before processing at the correlator (Solartron 1200, Solartron Instruments Ltd, U.K.) together with the modulation signal. The monochromator and correlator were controlled by a MINC $11 / 23$ instrumentation computer (DEC Ltd, U.K.).

To obtain cross-correlation spectrum, the monochromator was stepped through the wavelength range of interest, pausing to record the cross-correlation function at regular intervals. The cross-correlation functions were stored in the computer and corrected for the xenon lamp power spectrum. After the scan was completed the sheaf of correlation functions were plotred as a three-dimensional isometric surface.

The correlation imaging experiments were carried out using a thermal wave imaging system developed at UMIST. The source was a Krypton ion laser (Innova 90-K, Coherent UK Ltd, U.K.) modulated by an acousto-optic modulator (Mode1 304, Coherent UK Ltd, U.K.). The modulation signal was derived from an internal PRBS generator in the Solartron 1200. Scanning of the laser beam was carried out using orthogonally mounced scanning mirrors driven by feedback controlled galvanometer motors. After scanning the beam was focussed onto the surface of the sample contained in the gas-microphone cell. The correlator and laser scanning were controlled by the MINC 11/23. The cross-correlation function at each point on a line-scan across the surface of the sample was recorded and later plotted as a three-dimensional surface.

\section{RESULTS AND DISCUSSION}

Typical results from both spectroscopic and imaging experiments are presented. Figure 1 shows the correlation spectrum obtained from a bulk-dyed red polymer film of approximately lmm thickness. The wavelength range was between 300 and $600 \mathrm{~nm}$. The sample is thermally thick on the timescalc of the experiment. The absorption profile of the dye can be clearly seen, and the shape of the correlation function indicate that the light absorbing structures extend throughout the film thickness.

Figure 2 shows the correlation image of a line scan across an opaque sample with a sub-surface defect. The sample was prepared by drilling a $2 \mathrm{~mm}$ diameter hole through the face of a small piece of aluminium. The hole was filled with epoxy resin, polished flat and spray painted black to hide the defect. The image represents a line scan across the centre of the defect and back to solid metal. Initially the impulse response rises rapidly to a peak which is of approximately equal amplitude across the scan. This is due to the surface coating of paint which was evenly applied over the whole surface. After the initial response, two very different types of correlogram are obtained. Where the substrate is solid metal, the rcsponse decays rapidly as heat is conducted into the bulk of the substrate. Where the substrate is epoxy the response continues to build up to a peak and then decays slowly due to the much lower thermal diffusivity of the epoxy. The use of the correlation method allows information about the surface and subsurface characteristics of the sample to be obtained in the same experiment.

\section{REFERENCES}

1. A.Rosencwaig, "Photoacoustics and Photoacoustic Spectroscopy",
1980, J.Wiley and Sons.
A.Rosencwaig, "Scanned Image Microscopy", E. A.Ash (Ed.),
Academic Press, 1980, pp.291-317.
G.F.Kirkbright, R.M.Miller, Analyst, 1982, 107, 798-802.
3.
K.R.Godfrey, Automatica, 1980, 16, 527-534.
4. Y.Sugitani, A.Uejima, K.Kato, J.Photoacoustics, 1982, 1, 217.
5. G.F.Kirkbright, R.M.Miller, Anal.Chem., 1983., 55, 502-506.
6.
7.M.Ashworth, S.L.Castleden, G.F.Kirkbright and D.E.M.Spiliane,
J.Photoacoustics, 1982, 1, 151-160.

